\title{
Response Assessment Following Image-Guided Therapy of Hepatocellular Carcinoma
}

\author{
Pankaj Gupta ${ }^{1}$ Naveen Kalra ${ }^{1}$ Ajay Gulati ${ }^{1}$ Vishnu Dev ${ }^{1} \quad$ Ujjwal Gorsi ${ }^{1}$ Sreedhara B Cheluvashetty ${ }^{1}$ \\ Praveen Kumar-M² Ajay Duseja ${ }^{3} \quad$ Virendra Singh ${ }^{3} \quad$ Radha K. Dhiman $^{3}$ Manavjit Singh Sandhu ${ }^{1}$
}

1Department of Radiodiagnosis and Imaging, Postgraduate Institute of Medical Education and Research (PGIMER), Chandigarh, India

Address for correspondence Naveen Kalra, MD, Department of

${ }^{2}$ Department of Pharmacology, Postgraduate Institute of Medical Radiodiagnosis and Imaging, Postgraduate Institute of Medical Education Education and Research (PGIMER), Chandigarh, India

${ }^{3}$ Department of Hepatology, Postgraduate Institute of Medical

Education and Research (PGIMER), Chandigarh, India

and Research, Chandigarh, India (e-mail: navkal2004@yahoo.com).

J Clin Interv Radiol ISVIR:2020;4:88-97

\begin{abstract}
Image-guided locoregional therapies have an important role in the management of patients with hepatocellular carcinoma (HCC). Recent advances in the ablative as well as endovascular therapies have expanded the role of interventional radiologists in the treatment of HCC. Following image-guided therapy, an accurate response assess-

Keywords

- ablative therapies

- hepatocellular carcinoma

- response ment is vital. Knowledge regarding normal postprocedure changes and subtle signs of residual or recurrent disease is important. In this review, we discuss various response evaluation criteria currently employed for HCC. We also discuss the postprocedure imaging features suggestive of residual disease or recurrence and imaging biomarkers for response assessment.
\end{abstract}

\section{Introduction}

Hepatocellular carcinoma (HCC) is one of the most common cancers worldwide and its incidence has been increasing. It is ranked as the sixth most common cancer globally, with the highest rate seen in Eastern Asia and sub-Saharan Africa. It is the fourth most frequent cause of cancer-related death. ${ }^{1}$

In adults, HCC comprises almost $90 \%$ of the primary liver tumors and usually carries a grave prognosis, if not treated early. It is seen more commonly in men with a male to female ratio of $\sim 2$ to 2.5 : 1 . The majority ( $~ 90 \%)$ of cases of HCCs arise in the setting of cirrhosis and are associated with an identifiable underlying etiology. ${ }^{1}$ Approximately one-third of all cirrhotic patients may develop HCC in their lifetime. The frequent underlying causes are chronic viral hepatitis B caused by hepatitis B virus (HBV) and hepatitis C virus (HCV), chronic alcohol intake, and aflatoxin exposure. There is a decline in the number of HBV-related cases in endemic countries due to universal infant vaccination against HBV. Nonalcoholic steatohepatitis, on the other hand, is increasingly being identified as a cause of cirrhosis and HCC.

Diagnosis of HCC is based on a combination of imaging features and histopathology. In the setting of a cirrhotic liver, the diagnosis of HCC is based on the detection of vascular derangement typical of hepatic carcinogenesis observed on the contrast-enhanced imaging techniques. The European Association for the Study of Liver (EASL) and the American Association for the Study of the Liver Disease (AASLD) panels recommend the use of multiphasic contrast-enhanced computed tomography (CECT) and dynamic contrastenhanced magnetic resonance imaging (MRI) for the diagnosis of HCC. ${ }^{2}$ Contrast-enhanced ultrasound (CEUS) has been recommended by the EASL; however, AASLD does not include CEUS as a first-line test. The major features for the diagnosis of HCC as defined by the liver imaging reporting and data system (LI-RADS) classification system are the presence of arterial phase hyperenhancement (APHE), washout on the portal venous, or delayed phases with an enhancing capsule on the delayed phase. ${ }^{3}$ In the setting of a noncirrhotic liver, the imaging features of HCC are similar. However, as the specificity is lower, the diagnosis of HCC in this setting is confirmed at histopathology.

Various clinical staging systems have been proposed for prognostication and for guiding treatment in HCCs, most widely used being the Barcelona Clinic Liver Cancer (BCLC) staging system (-Table $\mathbf{1}){ }^{2}$ It comprises of prognostic published online July 1,2020
DOI https://doi.org/

10.1055/s-0040-1708579

ISSN 2457-0214.
(C)2020 by Indian Society of Vascular and Interventional Radiology
License terms

(ㅇ)( $\Theta \circledast$ 
Table 1 Various staging systems in hepatocellular carcinoma

\begin{tabular}{|l|l|}
\hline Pathological & Clinical \\
\hline $\begin{array}{l}\text { Liver Cancer Study Group of Japan staging } \\
\text { system }\end{array}$ & $\begin{array}{l}\text { Okuda staging } \\
\text { system }\end{array}$ \\
\hline Japanese Integrated Staging score & $\begin{array}{l}\text { Cancer of the } \\
\text { Liver Italian } \\
\text { Program score }\end{array}$ \\
\hline Chinese University Prognostic Index & $\begin{array}{l}\text { Barcelona Clinic } \\
\text { Liver Cancer } \\
\text { staging system }\end{array}$ \\
\hline $\begin{array}{l}\text { American Joint Committee on Cancer/Interna- } \\
\text { tional Union Against Cancer staging system }\end{array}$ & - \\
\hline
\end{tabular}

variables related to tumor status, liver function (measured by Child-Pugh score), and performance status. ${ }^{4}$

The mainstay of treatment in patients with very early HCC is surgery (hepatic resection or liver transplantation) and ablation. ${ }^{5}$ In early HCC not eligible for resection or transplant, ablation is indicated. ${ }^{6}$ For the intermediate stage, unresectable HCCs, transarterial chemoembolization (TACE) is the standard of care. Selective internal radiotherapy or transarterial radio-embolization (TARE) is not included in the BCLC algorithm but is recommended for patients with advanced HCC with main PV invasion. The success of various locoregional treatment (LRT) methods is assessed by serial imaging. Various classification systems have been proposed to evaluate patients after systemic or LRT. ${ }^{6}$ The size-based criteria include World Health Organization and Response Evaluation Criteria in Solid Tumors (RECIST). However, following treatment, the size of the lesion may not decrease or may in fact increase. The functional (enhancement based) criteria that rely on the changes in the enhancing component of the tumor are more useful. These include modified RECIST (mRECIST), EASL, Response Evaluation Criteria in the Cancer of the Liver, and Choi criteria. Of these, mRECIST is the most widely used. In a study by Forner et al, the response assessment following percutaneous ablation and TACE was assessed using RECIST and EASL criteria. The authors found that by employing RECIST, none of the patients achieved a complete response (CR) and $30.9 \%$ of patients had progressive disease (PD). When response was evaluated using EASL guidelines, $54.5 \%$ of patients achieved a CR and only $14.5 \%$ had PD. With the availability of new functional imaging methods including diffusion-weighted imaging (DWI) and perfusion imaging, these may allow the response assessment with greater accuracy.

Various imaging findings following LRT have been described in the literature, and this article aims to summarize and highlight the normal imaging features as well as findings suggestive of the residual or recurrent disease. A brief overview of the newer functional imaging biomarkers for assessing treatment response has also been provided.

\section{Imaging after LRT}

Imaging plays a vital role in the follow-up of patients with HCCs after LRT and helps in the assessment of treatment response and early detection of residual or recurrent tumors.
Imaging also helps assess early detection of complications. Multiphasic CECT or MRI is widely recommended for response assessment after LRT.

As already discussed, the size of the lesion may not decrease or may in fact increase. Thus functional (enhancement based) criteria depicting the changes in the enhancing component of the tumor are more reliable. The different types of responses according to the mRECIST criteria have been summarized in - Table 2. A new LI-RADS treatment response algorithm has also been proposed recently and is aimed at providing standardization in reporting (-Table 3). ${ }^{3}$-Fig. 1 highlights the various response evaluation criteria in HCC. The limitation of the enhancement-based criteria is the lack of validation for assessing HCC after TACE and ablative therapies. ${ }^{7}$ The evaluation of infiltrative lesions is difficult.

The imaging features following various LRT are broadly similar, with the treated area appearing hypoattenuating on follow-up CT with no postcontrast enhancement, suggestive of necrosis ( - Fig. 2, Table 4 ). The central coagulative necrosis may appear hyperdense on noncontrast CT and hyperintense on T1-weighted images (T1WIs). ${ }^{7} \mathrm{~T} 2$ hyperintensity is usually attributable to hemorrhage, liquefactive necrosis, or inflammation. Other ancillary imaging features have also been described and are specific to the treatment modality being used, and hence will be described under the respective subheadings. For most forms of the locoregional therapies, first follow-up imaging is performed after 4 weeks. Subsequently, imaging is recommended every 3 months for 2 years. ${ }^{8}$ In

Table 2 Assessment of target lesion response according to modified Response Evaluation Criteria in Solid Tumors criteria

\begin{tabular}{|l|l|}
\hline $\begin{array}{l}\text { Complete } \\
\text { response }\end{array}$ & $\begin{array}{l}\text { Disappearance of any intratumoral arterial } \\
\text { enhancement in all target lesions }\end{array}$ \\
\hline $\begin{array}{l}\text { Partial re- } \\
\text { sponse }\end{array}$ & $\begin{array}{l}\text { At least a } 30 \% \text { decrease in the sum of diam- } \\
\text { eters of viable (enhancement in the arterial } \\
\text { phase) target lesions }\end{array}$ \\
\hline Stable disease & $\begin{array}{l}\text { Any cases that do not qualify for either partial } \\
\text { response or progressive disease }\end{array}$ \\
\hline $\begin{array}{l}\text { Progressive } \\
\text { disease }\end{array}$ & $\begin{array}{l}\text { An increase of at least 20\% in the sum of the } \\
\text { diameters of viable (enhancing) target lesions }\end{array}$ \\
\hline
\end{tabular}

Table 3 LI-RADS response criteria

\begin{tabular}{|l|l|}
\hline $\begin{array}{l}\text { Response } \\
\text { category }\end{array}$ & Criteria \\
\hline $\begin{array}{l}\text { LR-TR } \\
\text { nonviable }\end{array}$ & $\begin{array}{l}\text { a. No lesional enhancement or } \\
\text { b. Treatment-specific expected enhancement } \\
\text { pattern }\end{array}$ \\
\hline $\begin{array}{l}\text { LR-TR } \\
\text { equivocal }\end{array}$ & $\begin{array}{l}\text { Enhancement pattern atypical for treatment- } \\
\text { specific expected enhancement pattern and not } \\
\text { meeting criteria for definitely or probably viable }\end{array}$ \\
\hline $\begin{array}{l}\text { LR-TR } \\
\text { viable }\end{array}$ & $\begin{array}{l}\text { Nodular, mass-like or thick irregular soft tissue } \\
\text { showing any of the following: } \\
\text { a. Arterial phase hyperenhancement } \\
\text { b. Washout appearance } \\
\text { C. Enhancement similar to pretreatment }\end{array}$ \\
\hline
\end{tabular}

Abbreviations: LI-RADS, liver imaging reporting and data system; LR-TR, locoregional-treatment response. 


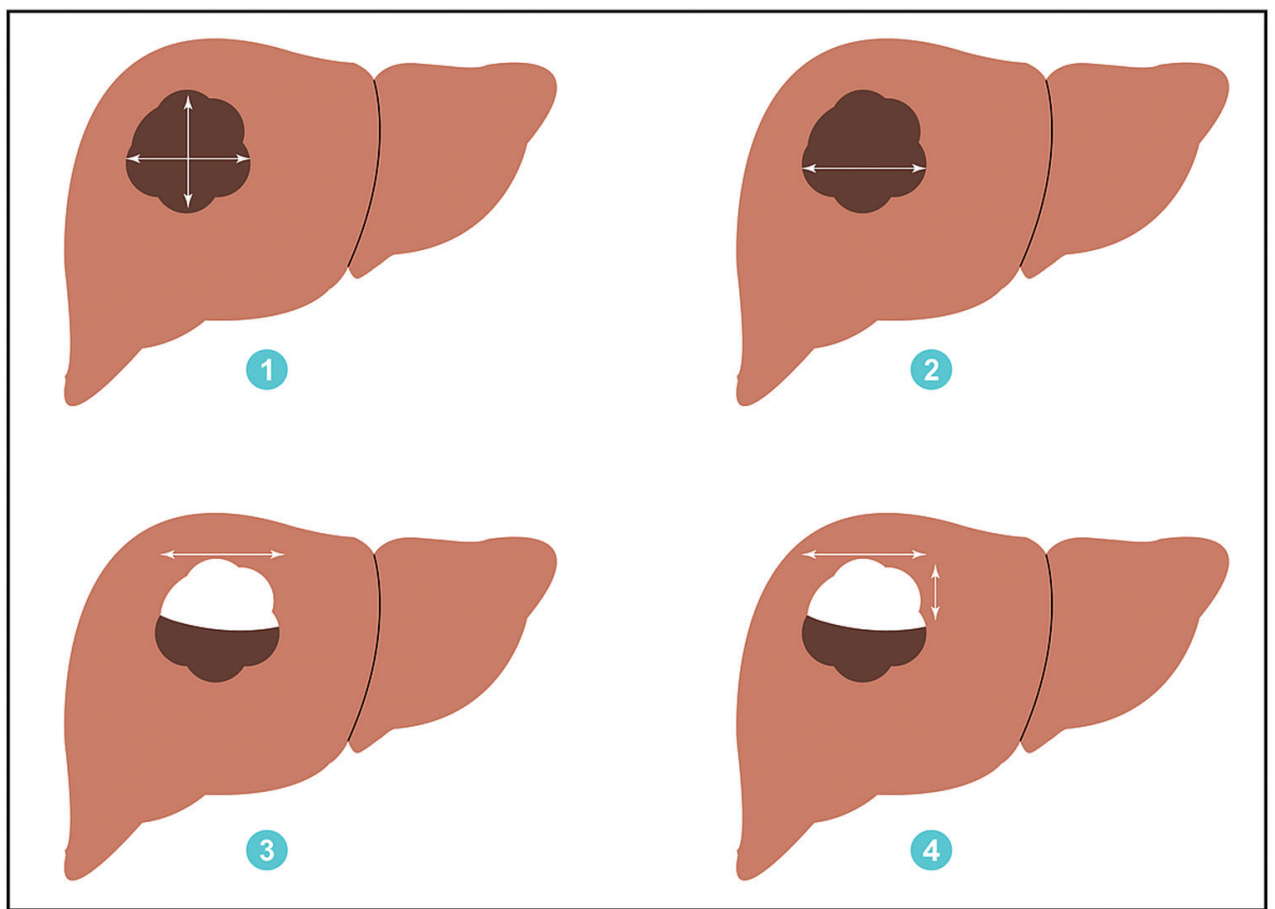

Fig. 1 Response evaluation criteria in hepatocellular carcinoma. 1. WHO criteria, 2. RECIST criteria, 3. mRECIST criteria, 4. EASL criteria. mRECIST, modified Response Evaluation Criteria in Solid Tumors; RECIST, Response Evaluation Criteria in Solid Tumors; WHO, World Health Organization;

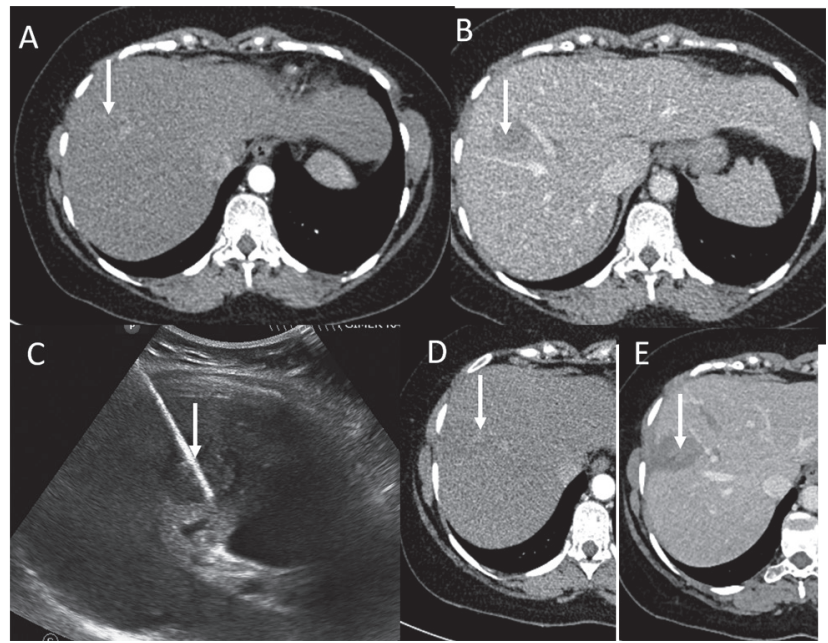

Fig. 2 Imaging features of complete response following interventional radiology treatment of hepatocellular carcinoma. (A) and (B) show an arterial enhancing nodule (arrow, $\mathbf{A}$ ) in segment VIII that shows washout in the venous phase (arrow, B). The lesion was treated with microwave ablation (MWA) (arrow, C). Imaging following MWA shows a subtle hypodense lesion that shows no arterial phase enhancement (arrow, D). On the venous phase, no thick rim of enhancement is seen (arrow, E).

patients treated with TARE, the first follow-up scan is usually deferred till 3 months following the therapy because of the delayed effects of TARE on tumor necrosis and shrinkage. ${ }^{9}$

\section{Transarterial Chemoembolization}

Following conventional TACE (cTACE), the high-density iodized oil usually stains the treated area for months,
Table 4 Findings on imaging following locoregional treatment of HCC

\begin{tabular}{|l|l|l|}
\hline $\begin{array}{l}\text { Locoregional } \\
\text { treatment }\end{array}$ & Normal finding & $\begin{array}{l}\text { Findings suggestive } \\
\text { of recurrence }\end{array}$ \\
\hline Ablation & $\begin{array}{l}\text { Smooth thin } \\
(<5 \text { mm }) \text { periph- } \\
\text { eral arterial rim } \\
\text { enhancement } \\
\text { Air within the lesion } \\
\text { Shunting around the } \\
\text { lesion }\end{array}$ & $\begin{array}{l}\text { Thick or irregular rim } \\
\text { enhancement } \\
\text { Rim enhancement in } \\
\text { arterial phase with } \\
\text { washout in venous or } \\
\text { delayed phase }\end{array}$ \\
\hline TACE & $\begin{array}{l}\text { Peritumoral } \\
\text { hypervascularity on } \\
\text { arterial phase }\end{array}$ & $\begin{array}{l}\text { Filling defect in lipiodol } \\
\text { uptake on NCCT } \\
\text { Areas of T2W hyper- } \\
\text { intensity within the } \\
\text { treated lesion } \\
\text { Areas of arterial } \\
\text { enhancement with } \\
\text { washout in venous phase } \\
\text { in the treated lesion }\end{array}$ \\
\hline TARE & $\begin{array}{l}\text { Heterogeneous } \\
\text { enhancement in } \\
\text { perivascular distribu- } \\
\text { tion in treated part }\end{array}$ & $\begin{array}{l}\text { Areas of arterial } \\
\text { enhancement with } \\
\text { washout in venous phase } \\
\text { in the treated lesion }\end{array}$ \\
\hline
\end{tabular}

Abbreviations: HCC, hepatocellular carcinoma; NCCT, noncontrast computed tomography; TACE, transarterial chemoembolization; TARE, transarterial radio-embolization.

appearing hyperattenuating on follow-up CTs, whereas the contrast used in drug-eluting bead (DEB)-TACE or bland embolization typically washes off after a few hours. ${ }^{10}$ In CTACE, the patients are usually imaged with a noncontrast CT within 24 to 48 hours following the procedure to evaluate the distribution of the iodized oil. The accumulation pattern of the iodized oil observed on CT has been classified 


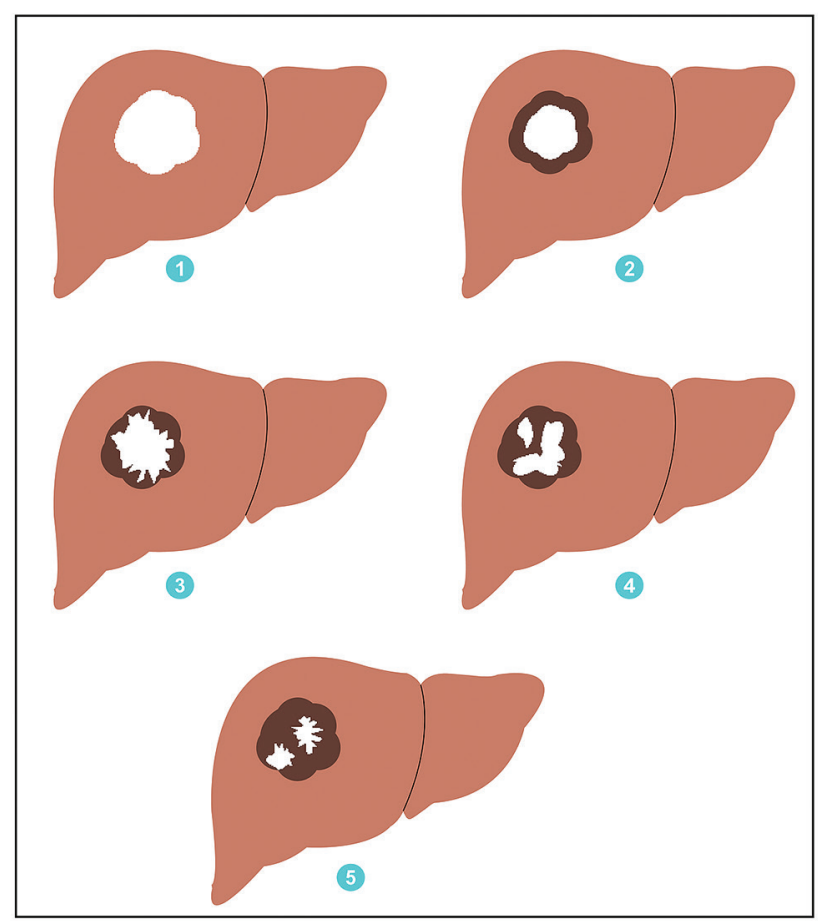

Fig. 3 Schematic diagram showing patterns of lipiodol uptake. 1. Type 1A, 2. Type 1B, 3. Type 2, 4. Type 3, 5. Type 4.

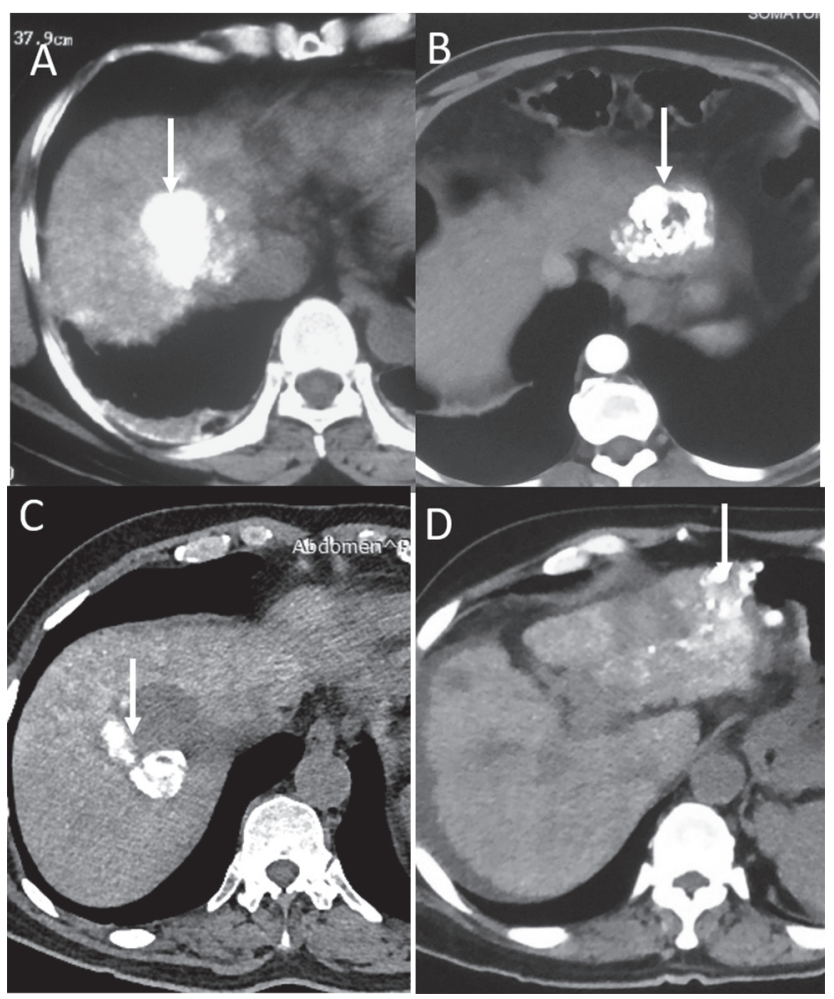

Fig. 4 Patterns of lipiodol uptake as depicted on computed tomography (CT). (A) Post-transarterial chemoembolization (TACE) noncontrast CT (NCCT) shows type 1 lipiodol uptake (arrow). (B) Post-TACE NCCT in another patient shows type 2 lipiodol uptake in segment II. (C) Noncontrast in another patient with hepatocellular carcinoma (HCC) in segment VIII shows type 3 lipiodol uptake. (D) Post-TACE NCCT in a patient with HCC involving left lobe shows type 4 lipiodol uptake. into four types ( $\boldsymbol{-}$ Figs. 3 and $\boldsymbol{- F i g s . ~} 4$ ).${ }^{11}$ Type 1 represents the homogenous accumulation of the iodized oil, with type $1 \mathrm{~A}$ showing accumulation even around the tumor and type $1 \mathrm{~B}$ is confined to the tumor area. In type II, there are partial defects in iodized oil accumulation within the tumor area. Faint accumulation of iodized oil is seen in type III and slight or no accumulation is noted in type IV. The distribution of iodized oil has a correlation with treatment response. Complete retention of iodized oil correlates with complete necrosis. ${ }^{12}$ Lack of uptake in the tumor may also be due to an aberrant arterial supply. ${ }^{12}$ CECT is done at 4 weeks and subsequently as per protocol described above. The treated necrosed area appears hypoattenuating on CT and hypointense on T2WI with no postcontrast enhancement. A thin rim of enhancement has been described around the treated lesion, which is likely due to reactive hyperemia in the early days and later due to inflammation. ${ }^{13}$ This peripheral rim of enhancement is usually thin ( $<5 \mathrm{~mm}$ in width), smooth, and shows persistent enhancement in the portal and delayed venous phases with no washout in these phases. ${ }^{13}$ It should not be confused with residual tumor, which generally appears as thick, nodular areas commonly at the edge of the treated area showing APHE with wash-

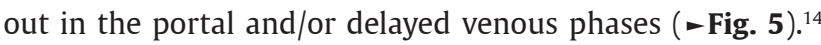
On MRI, the necrotic tissue appears hypointense on T2WI. The residual disease appears moderately hyperintense on $\mathrm{T} 2$ and shows postcontrast enhancement. For lesions showing predominantly coagulative necrosis or hemorrhage following TACE, the T1-weighted hyperintensity may lead to a difficulty in assessing postcontrast enhancement. In this context, subtraction images are beneficial. However, there should be no misregistration for the subtraction images. The hyperdensity of the iodized oil makes the evaluation of

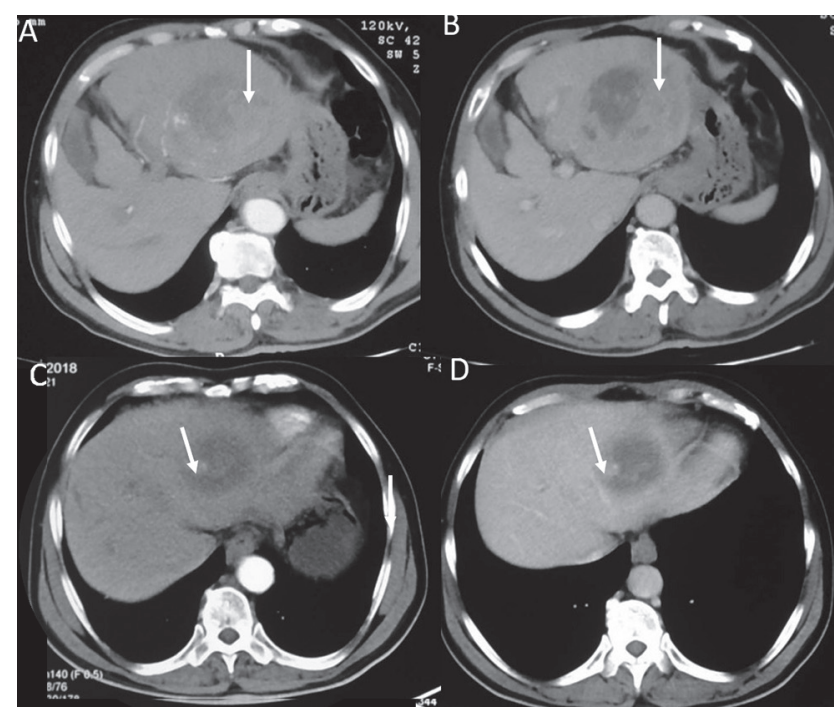

Fig. 5 Residual disease following transarterial chemoembolization (TACE). (A) Arterial phase image shows a large mass in left lobe showing thick peripheral enhancing component (arrow). (B) Venous phase image shows washout (arrow). (C) Computed tomography following drug-eluting bead-TACE (DEB-TACE) shows reduction in the size of the lesion as well as the enhancing peripheral component (arrow, C and D) suggestive of partial response. 
contrast enhancement challenging on CT. Iodized oil does not adversely affect the signal intensity on MRI. Thus, enhancement characteristics are better assessed on MRI, especially when subtraction images are assessed. However, both CT and MRI are equally accurate in assessment following DEB-TACE. In a study by Kloeckner et al, in the lipiodol TACE, CT underestimated the residual tumor. ${ }^{15}$ MRI in these cases was completely free of artifacts. ${ }^{4}$ Intratumoral gas containing areas have been reported after TACE. In a study by Bisseret et al comprising 286 procedures, intratumoral gas was demonstrated after 26 (9.1\%) TACE procedures. ${ }^{16}$ It was secondary to liver abscesses in only three patients. The factors that were significantly associated with the presence of intratumoral gas following TACE were larger mean baseline tumor diameter, DEB-TACE, and super-selective embolization. The presence of intratumor gas containing areas on follow-up CT scan was significantly associated with an objective treatment response. ${ }^{16}$

Imaging is a part of the "Assessment for Repeat Transarterial chemoembolization" (ART) criteria that guides the decision for repeat TACE. ${ }^{17}$ Radiological tumor response for ART is assessed according to the EASL criteria. The absence of tumor response is given a score of 1 . Other parameters included in ART are increase in the AST level by $25 \%$, and increase of Child-Pugh score of 1 . Patients with total score $\geq 2.5$ are not likely to benefit from repeat TACE. ${ }^{17}$

\section{Transarterial Radioembolization}

Imaging findings of patients with HCC on follow-up after TARE are like those following TACE. Diffuse increase in blood flow to the treated area has been described immediately following radioembolization that is observed as patchy or linear postcontrast enhancement within the lesion as well as an increased peritumoral enhancement on follow-up imaging shortly after the procedure that may persist up to 1 month and sometimes even later. ${ }^{7}$ This is because of the $\beta$ radiation emitted by the radioactive therapeutic agent termed as radiation effect. This enhancement diminishes gradually and disappears by $\sim 90$ days after treatment. Hence, the initial follow-up imaging after TARE is usually performed 6 to 12 weeks after therapy. ${ }^{7}$ As following other LRTs, presence of rim enhancement may be a normal finding after TARE. Lack of contrast enhancement on follow-up imaging can be considered a marker for successful treatment. Due to relative nontargeted nature of the treatment, the adjacent liver parenchyma surrounding the tumor may also be affected and may undergo fibrotic changes, observed on follow-up CT as an enhancement in the portal or delayed venous phases. ${ }^{18}$ TARE induces a delayed tumor necrosis and tumor shrinkage with a median time for these events reported to be 30 and 120 days, respectively.

\section{Ablative Therapies}

In general, the necrotic cavity following ablative procedure should exceed the original tumor margins by $\sim 5$ to $10 \mathrm{~mm}$ to include the potential microinvasion of the tumor. ${ }^{6}$ Also, the ablation zone may initially be larger due to edema, hemorrhage, and inflammatory changes following the procedure and these changes gradually subside over time. The necrotic cavity or the ablation zone is hypoattenuating on CT and does not show enhancement on postcontrast imaging. On MRI, it shows variable signal changes but lacks postcontrast enhancement. Another common finding on follow-up imaging after ablative therapies is a uniform thin peripheral rim enhancement that envelopes the entire ablation zone. This is considered a physiological response to thermal injury and has been reported in $79 \%$ of the cases. It has been reported to disappear by 1 month. The peripheral rim should be hyperto iso-attenuating on venous and delayed phase. Imaging is also critical in diagnosis of complications associated with ablative procedures like gastrointestinal bleeding, portal vein thrombosis, biliary injuries or leakage, liver abscesses, cholecystitis, or pleural effusion. ${ }^{19,20}$ Tumor seeding along the probe track is a rare complication, occurring in $\sim 0.2 \%$ of the cases and must be looked for carefully. Needle tract seeding is defined as the development of new neoplastic disease outside the liver capsule, either in the subcutaneous soft tissues or peritoneal cavity. It is seen as soft tissue with enhancement characteristics similar to the treated tumor.

\section{Radio Frequency Ablation (RFA), Microwave Ablation (MWA), and Cryoablation}

In addition to the common imaging findings described above, following thermal ablative techniques like RFA and MWA, a peripheral wedge-shaped arterial phase enhancement has been described adjacent to the ablation zone. This is due to the arterioportal shunts created by needle puncture or thermal injurry. ${ }^{7}$ This enhancement usually persists for about a month and vanishes gradually. It is also not uncommon to observe gas bubbles in the ablation zone and small fluid collections in the adjacent regions on imaging in immediate postablation period and should not be considered as signs of infection in the absence of clinical symptoms (- Fig. 6). ${ }^{21,22}$

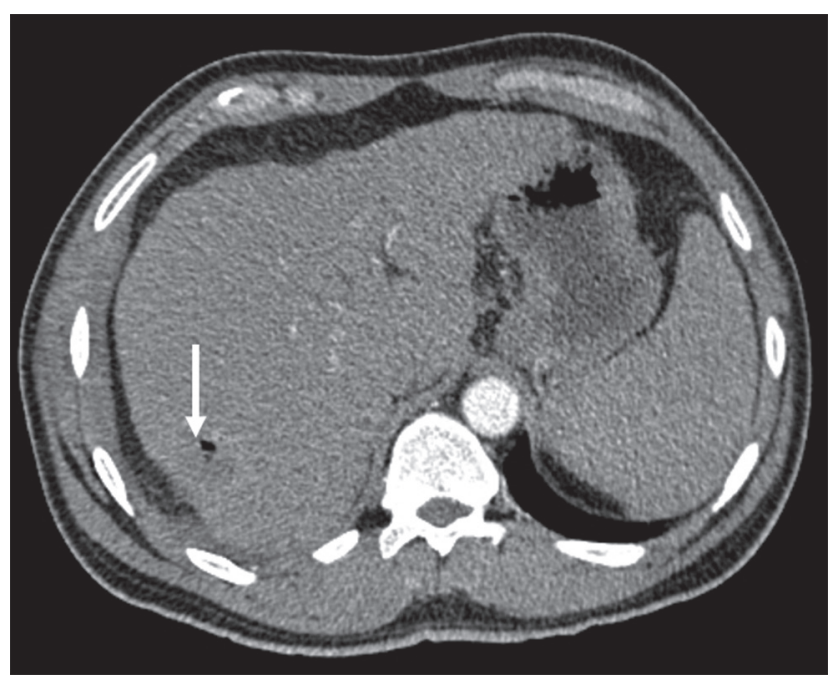

Fig. 6 Imaging following radiofrequency ablation. Computed tomography image shows small focus of air within the ablated lesion (arrow). There is no enhancement within the lesion or periphery in the arterial phase. 
The probe track is seen as a linear hypoattenuating structure on both T1WIs and T2WIs that are extended from the site of ablation up to the liver capsule. Other ancillary features that have been described include segmental intrahepatic biliary radicle dilatation and ipsilateral pleural effusion. The residual disease should be suspected in cases with an irregular thickening or new nodule usually observed along the periphery of the ablated area showing the APHE and washout in the venous or delayed phase ( - Fig. 7 ). ${ }^{23}$ The ablation zone gradually decreases in size over time and may disappear entirely or may be associated with focal hepatic atrophy or distortion of parenchymal architecture. Chiang et al studied the effect of MWA of HCC on hepatic arterial and venous vasculature. They reported occlusion of the portal vein, hepatic vein, and hepatic artery in the ablation zone in $39.7,15$, and $14.2 \%$ cases, respectively. There was a significant correlation between tumor progression and patent hepatic arteries within the ablation zone. However, the patency of portal vein and hepatic vein branches within the ablation zone did not predict local tumor progression. ${ }^{24}$ Shyn et al reported MRI findings predictive of tumor recurrence at 24 hours following cryoablation of liver tumor. They found that an ablative margin of less than $3 \mathrm{~mm}$ and a blood vessel bridging the tumor margin were present significantly more common in recurrent tumors. ${ }^{25}$

\section{Irreversible Electroporation}

Irreversible electroporation is a nonthermal ablation technique that is utilized for lesions located adjacent to vital structures. ${ }^{26}$ The peripheral rim of enhancement seen on imaging in the immediate postprocedural period likely represents the area of reversible electroporation and gradually reverts to normal over a period of time ( $\mathbf{- F i g . 8 )} .{ }^{27}$ As compared with thermal ablative techniques, blood vessels and biliary ductules are less vulnerable to damage during IRE, and it is not uncommon to observe these structures traversing through the ablated zone during follow-up scans. No studies have yet reported the response evaluation parameters unique to IRE.

\section{Advanced Imaging Biomarkers for Assessing Response after LRT}

\section{Dual-Energy CT}

Dual-energy CT (DECT) plays a role in the evaluation of response following LRTs in several ways. ${ }^{28,29}$ By generating virtual noncontrast (VNC) images, noncontrast CT acquisition may be avoided, thus reducing the radiation dose in patients undergoing serial follow-up imaging studies. DECT allows the identification of subtle hypervascularity and thus increased detection of hypervascular lesions. ${ }^{29}$ DECT has also been shown to improve the characterization of indeterminate sub-centimeter lesions by using iodine maps. Lee et al evaluated the role of DECT with VNC and iodine maps to evaluate therapeutic response to RFA in hepatic tumors. ${ }^{30}$ Seventy-five patients were assessed. The VNC images were rated as good as noncontrast computed tomography images in $90 \%$ of the patients and the iodine maps improved the conspicuity of the ablation margin due to higher contrast to noise ratio. In another recent study evaluating the role of DECT, Zhang et al evaluated the changes in volumetric iodine concentration (VIC) to predict response to MWA in rabbit intrahepatic VX2 tumor model. ${ }^{31}$ Among the various therapeutic response criteria, VIC on DECT was better at predicting response to MWA.

\section{Diffusion-Weighted Imaging}

Studies have demonstrated an increase in absolute apparent diffusion coefficient (ADC) values in responding lesions following TACE. ${ }^{14}$ Absolute ADC values were also shown to be useful in differentiating viable areas from necrotic areas following treatment. ${ }^{32,33}$ Besides, absolute ADC values also correlate well with the degree of necrosis. ${ }^{34}$ Recent research has shown DWI to be a potential imaging biomarker in early response assessment in patients of HCC after therapy. Kamel et al showed that there is a significant increase in ADC value 1 to 2 weeks after the first session of TACE. ${ }^{34}$ Favorable results regarding the use of DWI for prediction of tumor response have also been reported after TARE. ${ }^{35,36}$ In a study by Kamel et al, 19 HCCs in 13 patients were treated with yttrium-90-labeled microspheres. Following treatment, response evaluation was done using enhancement and ADC. There was a mean decrease in arterial enhancement of $22 \%$, a mean decrease in venous enhancement of $25 \%$, and a mean increase in ADC of $18 \% .^{36}$ In another study by Deng et al, six patients treated with ${ }^{90} \mathrm{Y}$ underwent evaluation with DWI. Tumor ADC increased significantly. ADC increased in all the lesions by a mean of $0.88 \times 10^{-3} \mathrm{~mm}^{2} / \mathrm{s}$, $42 \pm 16$ days after treatment $(p=0.004) \cdot{ }^{35}$ However, in the study by Goshima et al, DWI was not found to be a reliable method for the detection of local tumor recurrence compared

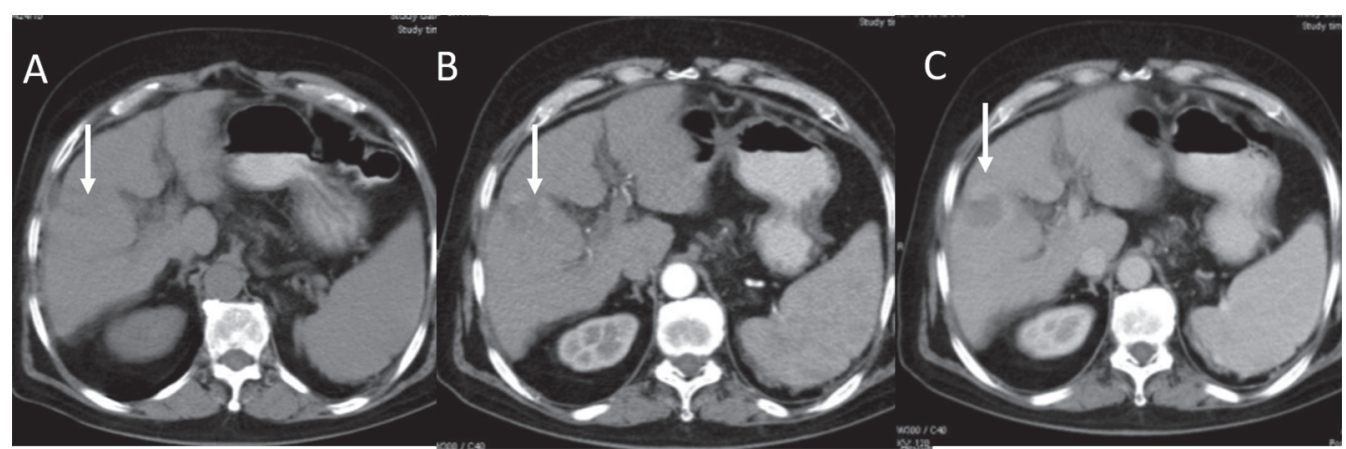

Fig. 7 Residual disease following radiofrequency ablation. (A) Noncontrast computed tomography shows a hypodense lesion with subtle internal hyperdensity (arrow). (B) The lesion shows subtle peripheral arterial enhancement (arrow). (C) There is a thick peripheral rim of enhancement in the venous phase (arrow). 


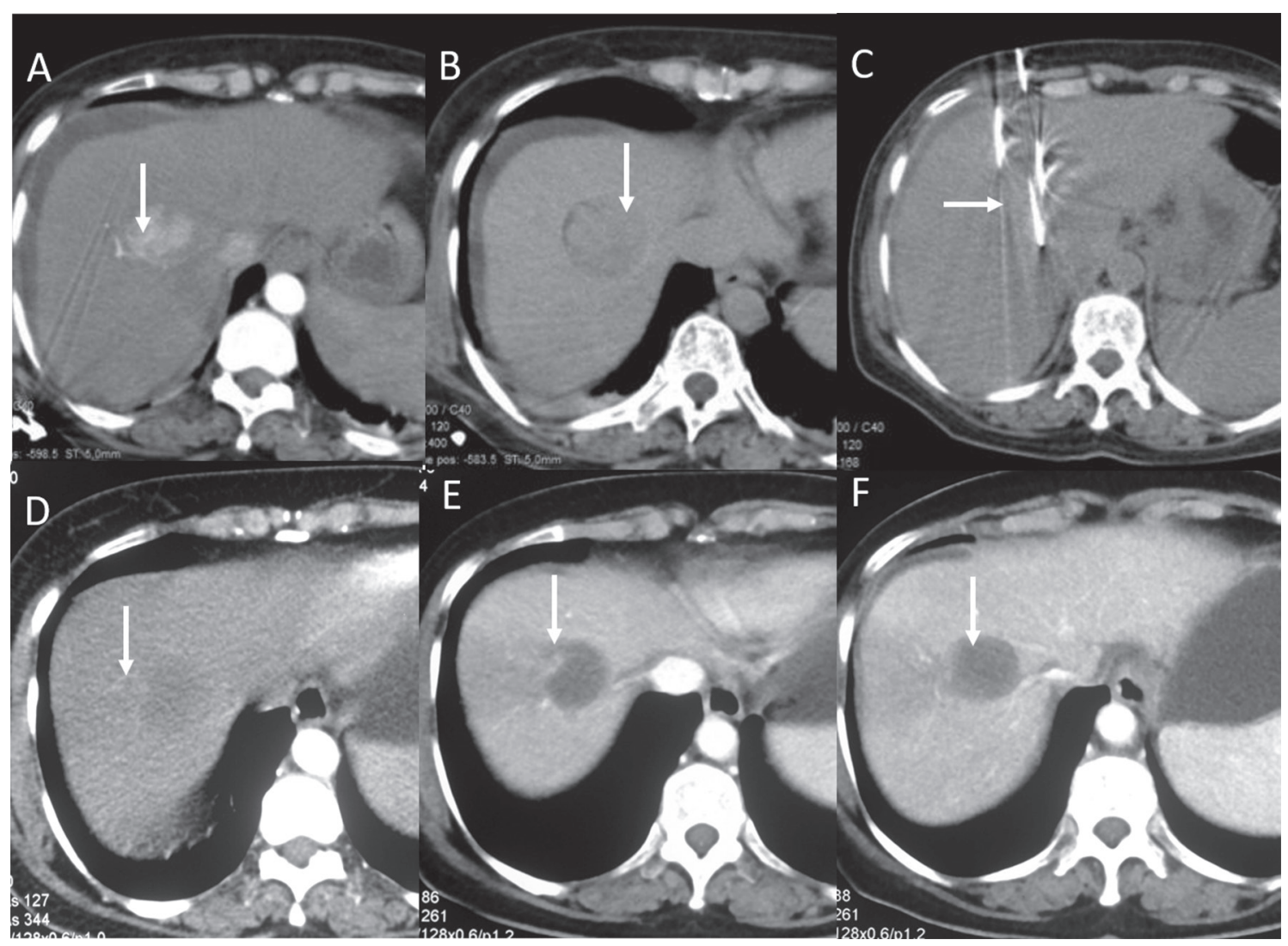

Fig. 8 Imaging after irreversible electroporation (IRE). (A) Arterial phase computed tomography (CT) shows arterial enhancing lesion in segment VIII. (B) There is washout in venous phase. An enhancing capsule is also seen (arrow). (C) IRE was performed using three probes (arrow). (D) Arterial phase image 4 weeks following IRE shows ill-defined arterial phase enhancement lateral to the lesion (arrow). (E) This area has a wedge-shaped configuration on venous phase CT (arrow), suggestive of a vascular phenomenon. (F) No abnormal lesional enhancement is seen (arrow).

with contrast-enhanced MRI. ${ }^{37}$ The role of pretreatment ADC in predicting response to TACE is not clear. ${ }^{38,39} \mathrm{~A}$ few studies have also reported the role of DWI in tumor response assessment following RFA. ${ }^{40}$ Bonekamp et al reported the use of a semiautomated software for the evaluation of early changes in post-treatment $A D C$ and enhancement features following TACE. ${ }^{41}$ In patients with contraindications to contrast injection or with equivocal postcontrast enhancement patterns, DWI is an effective alternative. ${ }^{42}$ However, susceptibility to artifacts and lack of reproducibility limit its widespread use.

\section{Perfusion Imaging}

The fundamental principle of perfusion imaging is the temporal changes in the tissue enhancement following the administration of an intravenous contrast material. Various parameters have been described to measure these changes, and the commonly used parameters include blood flow (BF), blood volume (BV), time to peak (TTP), and mean transit time (MTT). Studies have shown a significant reduction in hepatic arterial perfusion (AP) and total hepatic BV predictive of favorable treatment response. ${ }^{43-45}$ In the study by Chen et al, AP, hepatic arterial fraction, and BV reduced significantly on perfusion $\mathrm{CT}$ following TACE when compared with the pre-TACE values in patients with partial response (PR).44 On the other hand, these values showed a significant increase in the patients with PD. In another study by Zhu et al, the antiangiogenic activity of bevacizumab was evaluated using perfusion CT. Compared with baseline, there was a significant decrease in the $\mathrm{BF}, \mathrm{BV}$, and permeability surface area product and an increase in MTT were seen on days 10 to 12 following bevacizumab administration alone. Patients with PD had lower baseline MTT and a higher percent increase than those with stable disease or PR. ${ }^{46}$

In a study by Ippolito et al, 14 patients treated with RFA were evaluated with perfusion CT. The AP and hepatic perfusion index (HPI) were significantly higher in the residual lesion compared with the ablated lesion. ${ }^{47}$ In another study, Marquez et al evaluated 20 patients with liver lesions (10 metastases and 10 HCC) treated with RFA using perfusion CT immediately after RFA. The AP and HPI values were increased in incomplete responders. ${ }^{48}$

Although the initial results are promising, the lack of technical standardization, the requirement of additional software and the extra radiation, cost, and time make perfusion imaging more of a research tool in the current setting.

\section{Contrast-Enhanced Ultrasound}

After treatment, lack of APHE on CEUS was shown to correlate with complete necrosis on CT, whereas persistent APHE correlated with viable tumor. The recent European Federation of Societies for Ultrasound in Medicine and Biology guidelines recognize the importance of CEUS in early evaluation 
following ablation and as a guiding tool for immediate retreatment of residual disease. ${ }^{49}$ CEUS has been used for response evaluation following TACE. The advantage of CEUS is that it can be used immediately following the treatment. Additionally, the iodized oil does not hinder the interpretation of contrast enhancement. Liu et al reported that CEUS is more sensitive than multiphase $\mathrm{CT}$ for the detection of small residual tumors following TACE. ${ }^{50}$ Similar results were published in a recent meta-analysis. ${ }^{51}$ However, in a study comparing the accuracy of CEUS versus CECT in response assessment following RFA, CEUS was found to have a significantly lower sensitivity compared with CECT in detecting new lesions and local tumor progression. ${ }^{52}$ However, in another study, CEUS was found to yield results similar to $\mathrm{CT}$ and MRI in response assessment following thermal (RFA/MWA) ablation. Catalano et al in their large retrospective study demonstrated that the incorporation of CEUS in the imaging of HCC after ablation is feasible and allows lesser CT and MRI examinations to be performed. ${ }^{53}$ In the study by Kaufmann et al CEUS was shown to perform equally to volume perfusion $\mathrm{CT}$ in early response assessment to TACE in HCC. ${ }^{54}$ Zhan et al assessed the role of quantitative CEUS in response evaluation following microwave ablation of HCC and found that the largest tumor diameter and TTP were associated with overall survival in both univariate and multivariate analysis..$^{55}$

\section{Positron Emission Tomography (PET)-CT}

Recent studies have established the role of PET-CT in early prognostication and diagnosis of recurrent or residual disease in HCC. ${ }^{56-58}$ A PET-based response assessment criteria known as Positron Emission Tomography Response Criteria in Solid Tumors (PERCIST) has also been proposed. ${ }^{59}$ However, $18 \mathrm{~F}$-fluorodeoxyglucose (18F-FDG) is not specific for HCC and there is lack of data on the standardized uptake value (SUV) in predicting survival. Other tracers with higher sensitivity for detecting HCC like 18 fluorocholine are also being investigated. ${ }^{60}$ In a retrospective study comprising patients who underwent LRT for HCC (TACE, RFA, percutaneous ethanol ablation), 18F-FDG PET was performed 1 month following the treatment to assess the response. The authors concluded that visual assessment of the PET-CT may be more useful than the quantitative assessment. ${ }^{61}$ The role of 18F-FDG PET-CT in response evaluation following ${ }^{90} \mathrm{Y}$-TARE has been investigated. Patients were divided into PET positive and PET negative based on the FDG uptake on the baseline scan. PET positive cases were classified 4 weeks following the treatment into metabolic responders and nonresponders. The authors concluded that PET-negative patients had longer overall survival. In the PET-positive group, metabolic responders have a better overall survival. ${ }^{61}$ PET-CT has a role in the evaluation of extrahepatic metastases in HCC. In a study by Lee et al, 138 patients with HCC underwent both PETCT and conventional imaging modalities. ${ }^{62}$ The diagnostic performance of each imaging modality for extrahepatic metastases was evaluated. PET-CT was found useful for detection of lung metastases $>1 \mathrm{~cm}$ and bone metastases. The primary tumor characteristics that were associated with extrahepatic disease were a tumor size more than $5 \mathrm{~cm}$ and SUV uptake more than 3.4. ${ }^{62}$

\section{Radiomics}

Additionally, radiomics is increasingly being studied to assess the response to treatment in HCC. Kloth et al compared the performance of CT texture analysis (CTTA) with perfusion $\mathrm{CT}$ for predicting response in patients undergoing DEB-TACE. ${ }^{63}$ They found a significant correlation between CTTA and perfusion CT parameters. CTTA parameters were found to predict mid-term response following TACE. Kim et al evaluated the use of radiomic features for predicting the survival of patients undergoing TACE. ${ }^{64}$ In this study comprising 88 patients, 116 radiomic features were studies. The authors compared three models (radiomic based, clinical and combined) and found that the combined model was a better predictor of survival. In another study, Shan et al evaluated the peritumoral radiomics model to predict recurrence following resection or ablative therapies. ${ }^{65}$ A total of 156 patients were studied and the authors concluded that peritumoral radiomics models performed better than the tumoral radiomics model for predicting the recurrence.

Researchers are investigating various other novel functional imaging biomarkers in the early assessment of patients after LRT for HCC, including MR spectroscopy, MR elastography, growth kinetics, and integrated PET-MR. ${ }^{59,66}$ Though initial trials show promising results, research in this direction is still in its infancy, and it will take many years before these techniques are validated.

\section{Conclusion}

The imaging of patients with HCC following locoregional therapies is vital for the assessment of treatment success and early detection of complications and/or residual or recurrent disease requiring re-treatment. It is generally accomplished using CECT or MRI, although other novel imaging biomarkers are also currently being investigated. For radiologists, familiarity with the post-treatment imaging characteristics and their correct interpretation is crucial for assessing treatment response and for guiding future therapies.

\section{Conflict of Interest}

None.

Financial Disclosure

None.

\section{References}

1 GBD 2015 Mortality and Causes of Death Collaborators. Global, regional, and national life expectancy, all-cause mortality, and cause-specific mortality for 249 causes of death, 1980-2015: a systematic analysis for the Global Burden of Disease Study 2015. Lancet 2016;388(10053):1459-1544

2 European Association for the Study of the Liver. Electronic address, easloffice@easloffice.euEuropean Association for the Study of the Liver. Management of hepatocellular carcinoma. J Hepatol 2018;69(1):182-236 
3 Kielar A, Fowler KJ, Lewis S, et al. Locoregional therapies for hepatocellular carcinoma and the new LI-RADS treatment response algorithm. Abdom Radiol (NY) 2018;43(1):218-230

4 Kalra N, Gupta P, Chawla Y, Khandelwal N. Locoregional treatment for hepatocellular carcinoma: the best is yet to come. World J Radiol 2015;7(10):306-318

5 Gupta P, Kalra N, Shyamkumar NK, et al. Indian Society of Vascular and Interventional Radiology Expert Consensus Statements for Ablation in Hepatocellular Carcinoma: Part I. J Clin Interv Radiol ISVIR 2020. [in press]

6 Lencioni R, Llovet JM. Modified RECIST (mRECIST) assessment for hepatocellular carcinoma. Semin Liver Dis 2010;30(1):52-60

7 Yaghmai V, Besa C, Kim E, Gatlin JL, Siddiqui NA, Taouli B. Imaging assessment of hepatocellular carcinoma response to locoregional and systemic therapy. AJR Am J Roentgenol 2013;201(1):80-96

8 European Association For The Study Of The Liver European Organisation For Research And Treatment Of Cancer. EASLEORTC clinical practice guidelines: management of hepatocellular carcinoma. J Hepatol 2012;56(4):908-943

9 Keppke AL, Salem R, Reddy D, et al. Imaging of hepatocellular carcinoma after treatment with yttrium-90 microspheres. AJR Am J Roentgenol 2007;188(3):768-775

10 Kubota K, Hisa N, Nishikawa T, et al. Evaluation of hepatocellular carcinoma after treatment with transcatheter arterial chemoembolization: comparison of Lipiodol-CT, power Doppler sonography, and dynamic MRI. Abdom Imaging 2001;26(2):184-190

11 Lim HS, Jeong YY, Kang HK, Kim JK, Park JG. Imaging features of hepatocellular carcinoma after transcatheter arterial chemoembolization and radiofrequency ablation. AJR Am J Roentgenol 2006;187(4):W341-9

12 Takayasu K, Arii S, Matsuo N, et al. Comparison of CT findings with resected specimens after chemoembolization with iodized oil for hepatocellular carcinoma. AJR Am J Roentgenol 2000;175(3):699-704

13 Kim HC, Chung JW, Lee W, Jae HJ, Park JH. Recognizing extrahepatic collateral vessels that supply hepatocellular carcinoma to avoid complications of transcatheter arterial chemoembolization. Radiographics 2005;25(Suppl 1) :S25-S39

14 Young S, Taylor AJ, Sanghvi T. Post locoregional therapy treatment imaging in hepatocellular carcinoma patients: a literature-based review. J Clin Transl Hepatol 2018;6(2):189-197

15 Kloeckner R, Otto G, Biesterfeld S, Oberholzer K, Dueber C, Pitton MB. MDCT versus MRI assessment of tumor response after transarterial chemoembolization for the treatment of hepatocellular carcinoma. Cardiovasc Intervent Radiol 2010;33(3):532-540

16 Bisseret $\mathrm{D}$, Ronot M, Abdel-Rehim M, et al. Intratumoral gas in hepatocellular carcinoma following transarterial chemoembolization: associated factors and clinical impact. J Vasc Interv Radiol 2013;24(11):1623-1631

17 Sieghart W, Hucke F, Pinter M, et al. The ART of decision making: retreatment with transarterial chemoembolization in patients with hepatocellular carcinoma. Hepatology 2013;57(6):2261-2273

18 Semaan S, Makkar J, Lewis S, Chatterji M, Kim E, Taouli B. Imaging of hepatocellular carcinoma response after 90Y radioembolization. AJR Am J Roentgenol 2017;209(5):W263-W276

19 Dromain C, de Baere T, Elias D, et al. Hepatic tumors treated with percutaneous radio-frequency ablation: CT and MR imaging follow-up. Radiology 2002;223(1):255-262

20 Sironi S, Livraghi T, Meloni F. De Cobelli F, Ferrero C, Del Maschio A. Small hepatocellular carcinoma treated with percutaneous RF ablation: MR imaging follow-up. AJR Am J Roentgenol 1999;173(5):1225-1229
21 Kim YS, Rhim H, Lim HK. Imaging after radiofrequency ablation of hepatic tumors. Semin Ultrasound CT MR 2009;30(2):49-66

22 Park MH, Rhim H, Kim YS, Choi D, Lim HK, Lee WJ. Spectrum of CT findings after radiofrequency ablation of hepatic tumors. Radiographics 2008;28(2):379-390, discussion 390-392

23 Nakazawa T, Kokubu S, Shibuya A, et al. Radiofrequency ablation of hepatocellular carcinoma: correlation between local tumor progression after ablation and ablative margin. AJR Am J Roentgenol 2007;188(2):480-488

24 Chiang J, Cristescu M, Lee MH, et al. Effects of microwave ablation on arterial and venous vasculature after treatment of hepatocellular carcinoma. Radiology 2016;281(2):617-624

25 Shyn PB, Mauri G, Alencar RO, et al. Percutaneous imaging-guided cryoablation of liver tumors: predicting local progression on 24-hour MRI. AJR Am J Roentgenol 2014;203(2):W181-91

26 Kalra N, Gupta P, Gorsi U, et al. Irreversible electroporation for unresectable hepatocellular carcinoma: initial experience. Cardiovasc Intervent Radiol 2019;42(4):584-590

27 Padia SA, Johnson GE, Yeung RS, Park JO, Hippe DS, Kogut MJ. Irreversible electroporation in patients with hepatocellular carcinoma: immediate versus delayed findings at MR imaging. Radiology 2016;278(1):285-294

28 Zhang LJ, Peng J, Wu SY, et al. Liver virtual non-enhanced CT with dual-source, dual-energy CT: a preliminary study. Eur Radiol 2010;20(9):2257-2264

29 Altenbernd J, Heusner TA, Ringelstein A, Ladd SC, Forsting M, Antoch G. Dual-energy-CT of hypervascular liver lesions in patients with HCC: investigation of image quality and sensitivity. Eur Radiol 2011;21(4):738-743

30 Lee SH, Lee JM, Kim KW, et al. Dual-energy computed tomography to assess tumor response to hepatic radiofrequency ablation: potential diagnostic value of virtual noncontrast images and iodine maps. Invest Radiol 2011;46(2):77-84

31 Zhang L, Wang N, Mao J, et al. Dual-energy CT-derived volumetric iodine concentration for the assessment of therapeutic response after microwave ablation in a rabbit model with intrahepatic VX2 tumor. J Vasc Interv Radiol 2018;29(10):1455-1461

32 Mohamed AM, Louka AL, Ghazee AF. The role of diffusion weighted MR imaging in assessment of hepatocellular carcinoma after radiofrequency ablation. The Egyptian Journal of Hospital Medicine 2017;68:1339-1341

33 Mannelli L, Kim S, Hajdu CH, Babb JS, Clark TW, Taouli B. Assessment of tumor necrosis of hepatocellular carcinoma after chemoembolization: diffusion-weighted and contrast-enhanced MRI with histopathologic correlation of the explanted liver. AJR Am J Roentgenol 2009;193(4):1044-1052

34 Kamel IR, Liapi E, Reyes DK, Zahurak M, Bluemke DA, Geschwind JF. Unresectable hepatocellular carcinoma: serial early vascular and cellular changes after transarterial chemoembolization as detected with MR imaging. Radiology 2009;250(2):466-473

35 Deng J, Miller FH, Rhee TK, et al. Diffusion-weighted MR imaging for determination of hepatocellular carcinoma response to yttrium-90 radioembolization. J Vasc Interv Radiol 2006;17(7):1195-1200

36 Kamel IR, Reyes DK, Liapi E, Bluemke DA, Geschwind JF. Functional MR imaging assessment of tumor response after 90Y microsphere treatment in patients with unresectable hepatocellular carcinoma. J Vasc Interv Radiol 2007;18(1 Pt 1) : 49-56

37 Goshima S, Kanematsu M, Kondo H, et al. Evaluating local hepatocellular carcinoma recurrence post-transcatheter arterial chemoembolization: is diffusion-weighted MRI reliable as an indicator? J Magn Reson Imaging 2008;27(4):834-839

38 Yuan Z, Ye XD, Dong S, et al. Role of magnetic resonance diffusion-weighted imaging in evaluating response after 
chemoembolization of hepatocellular carcinoma. Eur J Radiol 2010;75(1):e9-e14

39 Mannelli L, Kim S, Hajdu CH, Babb JS, Taouli B. Serial diffusion-weighted MRI in patients with hepatocellular carcinoma: prediction and assessment of response to transarterial chemoembolization. Preliminary experience. Eur J Radiol 2013;82(4):577-582

40 Lu TL, Becce F, Bize P, Denys A, Meuli R, Schmidt S. Assessment of liver tumor response by high-field (3 T) MRI after radiofrequency ablation: short- and mid-term evolution of diffusion parameters within the ablation zone. Eur J Radiol 2012;81(9):e944-e950

41 Bonekamp S, Jolepalem P, Lazo M, Gulsun MA, Kiraly AP, Kamel IR. Hepatocellular carcinoma: response to TACE assessed with semiautomated volumetric and functional analysis of diffusion-weighted and contrast-enhanced MR imaging data. Radiology 2011;260(3):752-761

42 Ye XD, Yuan Z, Zhang J, Yuan Z. Radiological biomarkers for assessing response to locoregional therapies in hepatocellular carcinoma: From morphological to functional imaging (Review). (Review) Oncol Rep 2017;37(3):1337-1346

43 Tamandl D, Waneck F, Sieghart W, et al. Early response evaluation using CT-perfusion one day after transarterial chemoembolization for HCC predicts treatment response and long-term disease control. Eur J Radiol 2017;90:73-80

44 Chen G, Ma DQ, He W, Zhang BF, Zhao LQ. Computed tomography perfusion in evaluating the therapeutic effect of transarterial chemoembolization for hepatocellular carcinoma. World J Gastroenterol 2008;14(37):5738-5743

45 Rathmann N, Kara K, Budjan J, et al. Parenchymal liver blood volume and dynamic volume perfusion CT measurements of hepatocellular carcinoma in patients undergoing transarterial chemoembolization. Anticancer Res 2017;37(10):5681-5685

46 Zhu AX, Holalkere NS, Muzikansky A, Horgan K, Sahani DV. Early antiangiogenic activity of bevacizumab evaluated by computed tomography perfusion scan in patients with advanced hepatocellular carcinoma. Oncologist 2008;13(2):120-125

47 Ippolito D, Bonaffini PA, Ratti L, et al. Hepatocellular carcinoma treated with transarterial chemoembolization: dynamic perfusion-CT in the assessment of residual tumor. World J Gastroenterol 2010;16(47):5993-6000

48 Marquez HP, Puippe G, Mathew RP, Alkadhi H, Pfammatter T, Fischer MA. CT perfusion for early response evaluation of radiofrequency ablation of focal liver lesions: first experience. Cardiovasc Intervent Radiol 2017;40(1):90-98

49 Claudon M, Dietrich CF, Choi BI, et al; World Federation for Ultrasound in Medicine; European Federation of Societies for Ultrasound. Guidelines and good clinical practice recommendations for Contrast Enhanced Ultrasound (CEUS) in the liver - update 2012: A WFUMB-EFSUMB initiative in cooperation with representatives of AFSUMB, AIUM, ASUM, FLAUS and ICUS. Ultrasound Med Biol 2013;39(2):187-210

50 Liu M, Lin MX, Lu MD, et al. Comparison of contrast-enhanced ultrasound and contrast-enhanced computed tomography in evaluating the treatment response to transcatheter arterial chemoembolization of hepatocellular carcinoma using modified RECIST. Eur Radiol 2015;25(8):2502-2511

51 Zhong J, Su Z, Zhang Y, et al. Contrast-enhanced ultrasonography versus contrast-enhanced computed tomography for assessment of residual tumor from hepatocellular carcinoma treated with transarterial chemoembolization: a metaanalysis. J Ultrasound Med 2018;37(8):1881-1890

52 Zheng SG, Xu HX, Lu MD, et al. Role of contrast-enhanced ultrasound in follow-up assessment after ablation for hepatocellular carcinoma. World J Gastroenterol 2013;19(6):855-865
53 Catalano O, Izzo F, Vallone P, et al. Integrating contrastenhanced sonography in the follow-up algorithm of hepatocellular carcinoma treated with radiofrequency ablation: single cancer center experience. Acta Radiol 2015;56(2):133-142

54 Kaufmann S, Schulze M, Spira D, Horger M. Comparison of volume perfusion computed tomography and contrast-enhanced ultrasound for assessment of therapeutic effect of transarterial chemoembolization in patients with hepatocellular carcinoma: a preliminary report. Acta Radiol 2016;57(1):8-12

55 Zhan Y, Zhou F, Yu X, et al. Quantitative dynamic contrastenhanced ultrasound may help predict the outcome of hepatocellular carcinoma after microwave ablation. Int J Hyperthermia 2019;35(1):105-111

56 Young H, Baum R, Cremerius U, Herholz K, Hoekstra O, Lammertsma AA, Pruim J. , andPrice P; European Organization for Research and Treatment of Cancer (EORTC) PET Study Group, Measurement of clinical and subclinical tumour response using [18F]-fluorodeoxyglucose and positron emission tomography: Review and 1999 EORTC recommendations. Eur J Cancer 199;35:1773-1782

57 Kim SH, Won KS, Choi BW, et al. Usefulness of F-18 FDG PET/ $\mathrm{CT}$ in the evaluation of early treatment response after interventional therapy for hepatocellular carcinoma. Nucl Med Mol Imaging 2012;46(2):102-110

58 Jreige M, Mitsakis P, Van Der Gucht A, et al. 18F-FDG PET/CT predicts survival after90Y transarterial radioembolization in unresectable hepatocellular carcinoma. Eur J Nucl Med Mol Imaging 2017;44(7):1215-1222

59 Yuan Z, Li WT, Ye XD, Zhu HY, Peng WJ. Novel functional magnetic resonance imaging biomarkers for assessing response to therapy in hepatocellular carcinoma. Clin Transl Oncol 2014;16(7):599-605

60 Kwee SA, Sato MM, Kuang Y, et al. [18F]Fluorocholine PET/CT imaging of liver cancer: radiopathologic correlation with tissue phospholipid profiling. Mol Imaging Biol 2017;19(3):446-455

61 Yuan Z, Ye XD, Dong S, Xu LC, Xiao XS. Evaluation of early imaging response after chemoembolization of hepatocellular carcinoma by phosphorus-31 magnetic resonance spectroscopy-initial experience. J Vasc Interv Radiol 2011;22(8):1166-1173

62 Lee JE, Jang JY, Jeong SW, et al. Diagnostic value for extrahepatic metastases of hepatocellular carcinoma in positron emission tomography/computed tomography scan. World J Gastroenterol 2012;18(23):2979-2987

63 Kloth C, Thaiss WM, Kärgel R, et al. Evaluation of texture analysis parameter for response prediction in patients with hepatocellular carcinoma undergoing drug-eluting bead transarterial chemoembolization (DEB-TACE) using biphasic contrast-enhanced CT image data: correlation with liver perfusion CT. Acad Radiol 2017;24(11):1352-1363

64 Kim J, Choi SJ, Lee SH, Lee HY, Park H. Predicting survival using pretreatment CT for patients with hepatocellular carcinoma treated with transarterial chemoembolization: comparison of models using radiomics. AJR Am J Roentgenol 2018;211(5):1026-1034

65 Shan QY, Hu HT, Feng ST, et al. CT-based peritumoral radiomics signatures to predict early recurrence in hepatocellular carcinoma after curative tumor resection or ablation. Cancer Imaging 2019;19(1):11

66 Donati OF, Hany TF, Reiner CS, et al. Value of retrospective fusion of PET and MR images in detection of hepatic metastases: comparison with 18F-FDG PET/CT and Gd-EOB-DTPAenhanced MRI. J Nucl Med 2010;51(5):692-699 